\title{
Residual Current Measurement using Helmholtz Coil Configuration with different Current Flow
}

\author{
Erwin Sutanto ${ }^{1}$, Frangky Chandra ${ }^{2}$, Eduardo Gonnelli ${ }^{3}$, Suhariningsih ${ }^{4}$ \\ ${ }^{1,2}$ Biomedical Engineering, Faculty of Science and Technology, UniversitasAirlangga, Indonesia \\ ${ }^{3}$ Nuclear and Energy Research Institute, IPEN/CNEN, Brazil \\ ${ }^{4}$ Physics Department, Faculty of Science and Technology, UniversitasAirlangga, Indonesia
}

\begin{tabular}{l} 
Article Info \\
\hline Article history: \\
Received Jan 13, 2018 \\
Revised Mar 10, 2018 \\
Accepted Mar 24, 2018 \\
\hline
\end{tabular}

Keyword:

Electrical safety devices Helmholtz coil configuration RCD (residual current device) Residual current measurement

\section{Corresponding Author:}

Erwin Sutanto,

Biomedical Engineering, Faculty of Science and Technology,

Universitas Airlangga,

Kampus C Unair, Surabaya 60115, Jawa Timur, Indonesia.

Email: erwin_sutanto@fst.unair.ac.id

\begin{abstract}
For leakage current safety, Residual Current Device (RCD) has been well known. The purpose of this work is to make the employment of low price components to measure residual current feasible and the Residual Current Device (RCD) must to be taken into account because it is a well known device for leakage current safety. For this purpose, experiments employing the Helmholtz Coil Configuration were performed with the different current flow. Furthermore, the residual current was formulated and simulated through the software Easy Java Simulation (EJS). The results showed that it is possible to move the magnet into different angles using leakage current with linear gradient as low as 0.382 degree/mA. Finally, it was proposed a way to increase the sensitivity and to reduce the hysteresis phenomenon.
\end{abstract}

Copyright $@ 2018$ Institute of Advanced Engineering and Science. All rights reserved.

\section{INTRODUCTION}

The increasing usage of medical equipments creates basic things to be taken care. One of them is how to assure the patient's safety. It is common to see the high voltage power supply for these medical equipments so the electrical safety has to be concerned. One of concerns is to avoid deathly electrical shocks. As similar like in industry, as common as overhead power transmission lines and pipelines, could have induced voltages that present a risk of electric shock to the operator safety [1]. This is actually can be avoided by having corect grounding [2], or by other means. They will prevent electrical shock's leakage current that endanger patient's life. One practical way to prevent the electrical shocks is by limiting the leakage current from the medical equipment. To do that, the Residual Current Device (RCD) can be used in combination with normal Miniature Circuit Breaker (MCB) which is already in common compared to the RCD. It is slightly different compared to MCB. The RCD is limiting the residual current.

RCD is only an application of the leakage current measurement. It is to trip the electrical power supply on certain amount of leakage current [3]. Typical RCD circuit is shown at Figure 1. In hospitals, not all medical equipments are equiped with RCD. In replacement, they are checked by the engineers periodically by measuring its leakage current value. It is usually measured using Multi-function Electrical Safety Tester [4]. One of possible reason may due to th cost of the RCD.

Looking details at RCD, the uncommon component is the toroid RCD. The other components are actually only coils that apply physics, the Faraday Law. The concept of using two coils which applying Faraday Law can also be seen at Electromagnetic Force-Displacement Sensor [5]. The other advanced application can be seen from Energy Transfer by Resonance Coupling [6]. The formulated configuration of using two coils is established with the Helmholtz Coil. Helmholtz Coil configuration is structured by two N- 
turn circular coils with radius $\mathrm{R}$, each perpendicular to the $\mathrm{y}$ axis symmetrically, with a distance $\mathrm{D}$. The original Helmholtz coil creates uniform magnetic field by having similar current flow direction on both circular coils. It will make similar magnetic field goes to the same direction. The application is well known for experiments in which need to have uniform Magnetic Field. The proposed residual current measurement, presents the same physical principal that the wide use of Helmholtz Coil.

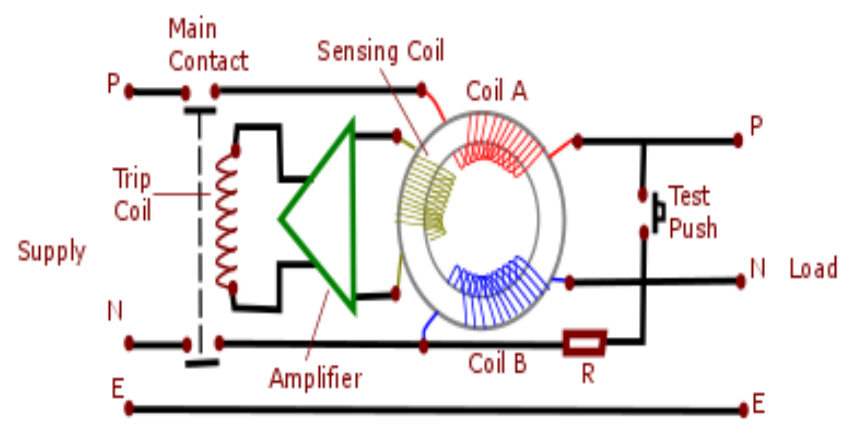

Figure 1. Typical RCD circuit

In this paper, it was proposed and developed a residual current device with low cost components. The first approach was considered using well-known formulas to compare the Helmholtz Coil and the common RCD. Furthermore, the magnetic field simulation of the experiment was performed employing the common open source software EJS. This software has been used for many physics education purposes [7]. In this work it was employed for sensitivity measurement [8]. Finally, it also tries to increase the instrument's sensitivity by doing a simple test. This work showed a common characteristic of measurement. It is like the hysteresis of magnetic field. That is why increasing number coil could be one of possible solution to realize a practical measurement. Target sensitivity is similar with typical RCD which is with possibility to limit $30 \mathrm{~mA}$. It is following IEC 60364-4-41 for protection against electrical shock [9].

\section{HELMHOLTZ COIL CONFIGURATION WITH DIFFERENT CURRENT FLOW}

In the configuration of this work, there is the same structure with normal Helmholtz Coil but it has different current flow. The idea is following the RCD principle which differentiating the magnetic fields from both circular coils.

\subsection{RCD}

Residual Current Device (RCD) is a safety device which is slightly different compared with the Miniature Circuit Breaker (MCB). If MCB is used to trip of over current load, this RCD is used to trip of over residual current. This difference is also seen at the limit current value. For MCB, a common current limit is about 6A [10]. For residual current, the common nominal is only about $30 \mathrm{~mA}$ [9]. The MCB normally will make sure the power distribution on each power network while RCD will be more responsible for the safety each on its different application. RCD in households and commercial enterprises will protect the building from possible fire because of special risk or hazard, like human life safety. RCD is widely used both at homes and in companies. This device is used as an additional protection against electric shock. The $\mathrm{RCD}$ is capable to detect over residual current directly.

The RCD consists of a toroid, two coils (Coil A and Coil B), and one reader coil (Sensing Coil) as its main component. In addition, the RCD commonly also includes an additional circuit to test the mechanism for current trip. The last part is a relay that serves to break the voltage as part of trip mechanism. Sometimes the relay is accompanied with an amplifier besides a trip coil. However, RCD is basically depending on the material that constitutes the Toroid to produce sufficient output from the RCD. A good Toroid material will have high permeability as required [11]. Then it can then activate the RCD relay with a relatively small current in mA.The overall mechanism to activate trip coil is shown in Figure 12.

Through the leakage current in the power grid, there are actually two possible path of how electric current flows. It is because when there is a leakage, the current will return to Supply via Ground. If compared with the normal path, then this is different. Under normal conditions, the current will return to Supply via Toroid's Coil B. It is then returning back to Suply N. Assuming that the current through Toroid's Coil A is $\mathrm{I}_{1}$ 
and the current through Toroid's Coil $\mathrm{B}$ is $\mathrm{I}_{2}$, the above statement can be rewritten as follows:

a. When the condition is normal:

$$
I_{1}=I_{2}
$$

b. When there is a leakage:

$$
I_{\text {leakage }}=I_{1}-I_{2}
$$

From the two current values that have been formulated above, the leakage current value will be read employing sensing coil. This coil will read electromotive force (e.m.f.) induction when a leakage current occurs. This phenomenon occurs when the current on Toroid's Coil A is not the same as the current on Toroid's Coil B. In physics, the leaking current will induces a magnetic flux in the toroid's magnetic core. Then that magnetic flux will be read by the sensing coil that is also wrapped on the Toroid core.

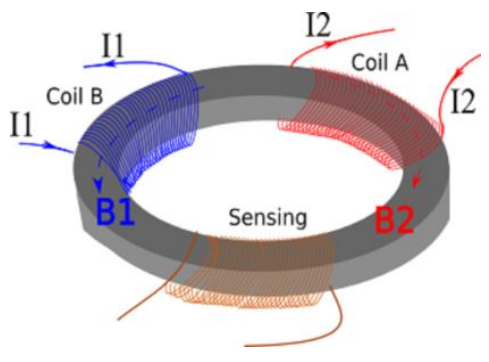

Figure 2. RCD Toroid's magnetic field

\subsubsection{Ampere law of RCD}

As previously discussed, the magnetic field value depends on the toroid material used. The material will define the variable of magnetic permeability, $\mu$. In addition, there are also turns's number of Coil A and Coil B, the medium or circumference of ds1, the small part of toroid's radius commonly expressed in $\theta 1$ radians, and the last radius $\mathrm{r}$ as the distance from the center of the circle which define the magnetic field. Assuming that there is no missing magnetic field from the medium, with the same number of turns on Coil A and Coil $\mathrm{B}, \mathrm{N}_{1}=\mathrm{N}_{2}=\mathrm{N}$, and $\theta_{1}=\theta_{2}=\theta$, the magnetic field difference resulting from the current leakage could be calculated by (3). Then by using (2), it is possible to rewrite the equation in leakage current term, $\mathrm{I}_{\text {leakage }}$. The final equation is shown below [8]:

$$
\Delta B=B_{1}-B_{2}=\frac{\mu_{r} N}{\theta . r}\left(I_{1}-I_{2}\right)=\frac{\mu_{r} N}{\theta . r} I_{\text {leakage }}
$$

\subsection{Comparison betweens RCD and helmholtz coil with different current flow}

The fundamental difference between the RCD and the Helmholtz Coil Configuration is attributed to the medium in which the magnetic field spreads. The RCD uses a toroid shaped medium and depends on its magnetic permeability. The required medium is a special material that has magnetic characteristic, while Helmholtz Coil does not need any. Thus Helmholtz Coil is simpler than RCD. It will make residual current measurements using this method more realistic. It is by only using low cost components, such as Coil or the electric wire.

On the other hand, there are similarities with the Different Current Flow between the RCD and Helmholtz Coil. Both of them use different currents principle. It is used to generate magnetic fields that will be opposite from each coils. For that, here will be re-formulated Magnetic Field equations resulting from leakage current.

\subsubsection{Biot savart law of helmholtz coil with different current flow}

The purpose of using Helmholtz Coil Configuration is to follow from the previous RCD mechanism. That is to obtain the difference of magnetic field value for two coils, B1 and B2. As it can be seen from Figure 3, the direction of the magnetic field from each solenoid is opposite on parallel axis, Y axis. 


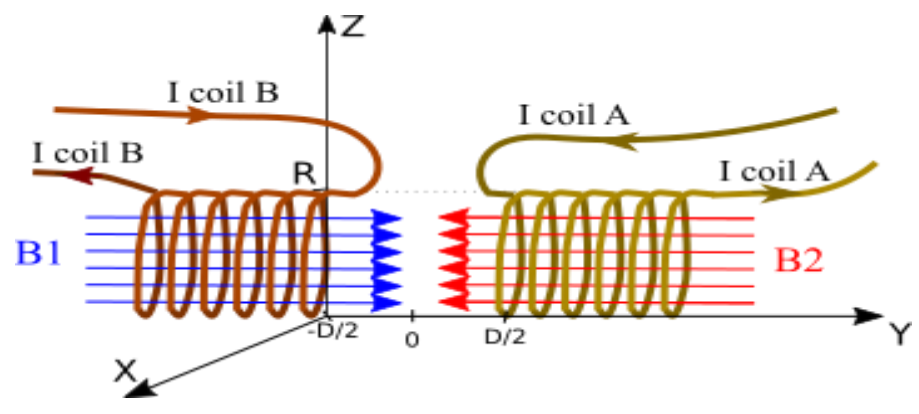

Figure 3. Helmholts coil with differtet current flow

To calculate the magnetic field difference, the magnetic field formula of one solenoid is required, where $\mu_{0}$ is the permeability of free space, $4 \pi \cdot 10-74 \pi \cdot 10^{-7}(\mathrm{~T} . \mathrm{m} / \mathrm{A})$. The magnetic field B of the solenoid can be obtained by using the Biot-Savart law. That is first by using a discrete approach. Then continue it using an Integral to obtain the total value of the total magnetic field, detonated by B [12]:

$$
B(z)=\frac{\mu_{0}}{4 \pi} \frac{I R^{2}(2 \pi)}{\left(R^{2}+z^{2}\right)^{3 / 2}}=\frac{\mu_{0} I R^{2}}{2\left(R^{2}+z^{2}\right)^{3 / 2}}
$$

From the magnetic field equations in which generated by one solenoid (4), the superposition principle can be used to obtain the difference between magnetic field from the two coils. The resulting equation can then be simplified by following the basic principles of Helmholtz Coil configuration. That is, the distance between two Coils A and Coil B is equal to the radius of each coil (R).

The simplification resulted in a magnetic field value that depends on the number of coil rotations $\left(\mathrm{N}_{\mathrm{A}}\right.$ and $\mathrm{N}_{\mathrm{B}}$ ), and the value of current in each coil ( $\mathrm{I}_{\text {Coil A }}$ and $\left.\mathrm{I}_{\text {Coil B }}\right)$. Finally, by equalizing the number of loops in each Coil $(\mathrm{N})$, the relationship between the magnetic field and the leakage current can be obtained [8]. That is, by applying (2) to (4) for leakage current, $\mathrm{I}_{\text {leakage. The Equation (5) was obtained: }}$

$$
\Delta B=\left(\frac{4}{5}\right)^{3 / 2} \frac{\mu_{0} N I_{\text {leakage }}}{2 R}
$$

\subsubsection{Magnetic field simulation}

By using the open source software, EJS, the above equations can be easily simulated. The simulation of this magnetic field difference is a modification of Helmholtz Coil [13]. It is made by subtracting the value of the magnetic field generated from one coil to another.

Through this simulation, the resulting magnetic field can be ascertained visually. It is useful to confirm which tool could be used later to read the generated magnetic field. It is also related with the way that design of residual current measurements would be when applied to medical equipments.

There are four inputs for the simulation program. The input is a component that has been described in (5). Among others are coil radius (R), number of coil rotation $(\mathrm{N})$, and current value on each coil (I Coil A and $\mathrm{I}_{\text {Coil B }}$ ).

Figure 4 shows the view of the program. The display shows the magnetic field difference conditions in the Helmholtz Coil configuration with $\mathrm{R}=50, \mathrm{~N}=1$, and no leakage current. This can be seen from the same value of $\mathrm{I}_{\text {CoilA }}$ and $\mathrm{I}_{\text {CoilB }}$. The program employs numerical methods for the calculation step. This simulation shows a good visualization and can describe the strength of the magnetic field difference in one Helmholtz Coil with Different Current Flow. 


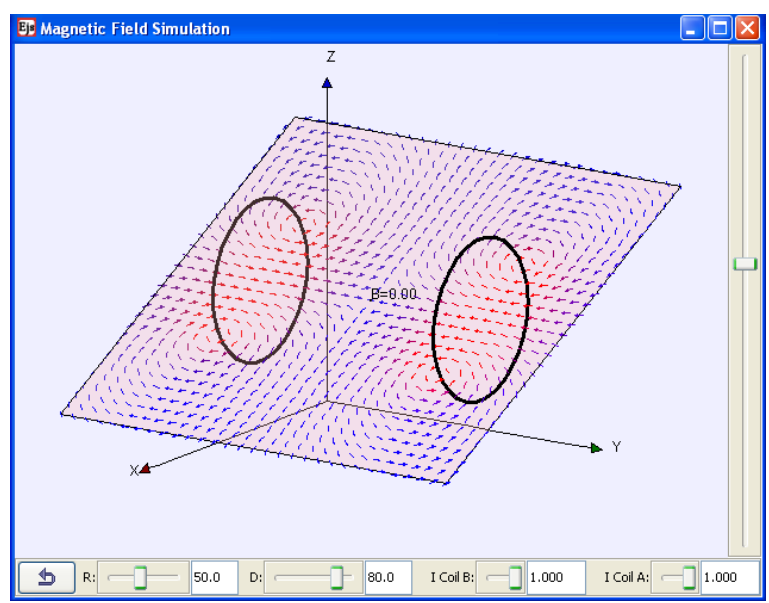

Figure 4. Magnetic field simulation

\section{EXPERIMENT WITH HELMHOLTZ COIL}

A simple device was built to have a real experiment for Helmholtz Coil Configuration. The device was consisted of two solenoid coils and a round shape neodymium strong permanent magnet in the middle. The permanent magnet was positioned in parallel to the coil axis and operated as a sensitive sensor [14]. The experiment is done by applying different Amperes values at each individual solenoid of the device. Then the leakage current was the difference of those two current values. From this experiment sensitivity of the possible leakage current could be found.

The permanent magnet should respond the magnetic field resulted from the leakage current described at (5). However, as a normal compass, this device has default orientation which is pointing to north of the Earth's magnetic field. It is also becoming the zero position of no current leakage flow. The Earth's magnetic field can also be used to confirm that there is no other force or frictions that affect its movement. It can be confirmed by having its pointing direction kept in one direction although the device is rotated.

The device is shown at Figure 5. It is a commercial Helmholtz Coil from Leybold [15]. It presents coils with 130 turns of winding, $150 \mathrm{~mm}$ of radius and there is the same distance between the two coils. This commercial apparatus comes with current source power supply. By using two power supply units, it was possible on simulated the different current value flowing into each coil. Thus it was possible on simulated different leakage current values by having the difference between the two current values at each coil.

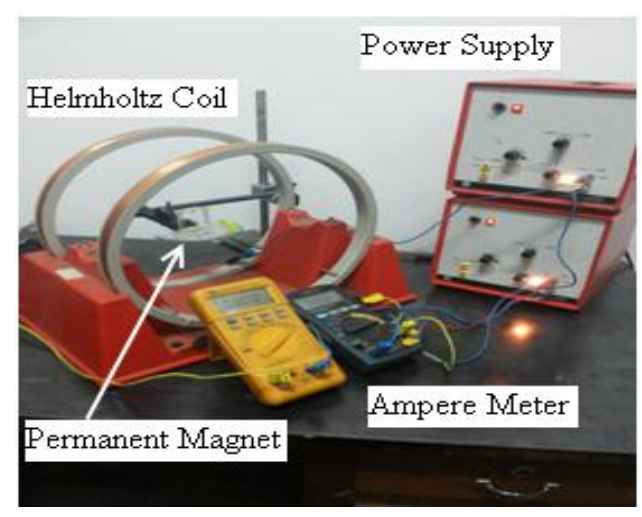

Figure 5. Helmholtz coil with different current flow experiment

\subsection{Residual current measurement}

This experiment showed good result in which can move the permanent magnet into different angles. Table1 shows the combination between (compass) permanent magnet deflection angle and its rated leakage current value from the experiment. 
The rated leakage current is the average from variations of differences value combination that flow at each individual coils. The compass deflection at 10 degree can be resulted from various combination of current flowing at Coil $\mathrm{A}$ and Coil B, for example; 0mA vs $24.7 \mathrm{~mA}$; $0.1 \mathrm{~mA}$ vs $24.8 \mathrm{~mA}$; or $46.7 \mathrm{~mA}$ vs 20.0mA; etc. Similar case was for all deflection degree. This leakage current was the simulation of possible residual current that may come from the combination of current flow at coil A and coil B.

Table1. Compassrotation VS LeakageCurrent

\begin{tabular}{cc}
\hline Deflection (degree) & Rated Leakage Current $(\mathrm{mA})$ \\
\hline-50 & -132.3 \\
-40 & -106.2 \\
-30 & -80.0 \\
-20 & -53.8 \\
-10 & -27.6 \\
0 & -1.5 \\
10 & 24.7 \\
20 & 50.9 \\
30 & 77.1 \\
40 & 103.3 \\
50 & 129.4 \\
\hline
\end{tabular}

Then the complete experimental data were plotted as shown in Figure 6. It is an $\mathrm{x}-\mathrm{y}$ plot from the experimental data. The data showed some variations of deflection for a single current difference between coil $\mathrm{A}$ and coil B. For the same current difference at $30 \mathrm{~mA}$ or 0.03 A between Coil A and Coil B, the deflection degree can be vary as: 40,41 , and 42 . The linear regression equation showed good linearity relationship between the deflection angle and leakage current. $\mathrm{R}^{2}$ parameter from fitting algorithm of Least Square method shows as high as 93\%. It showed that the equation is fits with the data. The linear equation is showing sensitivity from the equation gradient, that is 0.382 degree $/ \mathrm{mA}$.

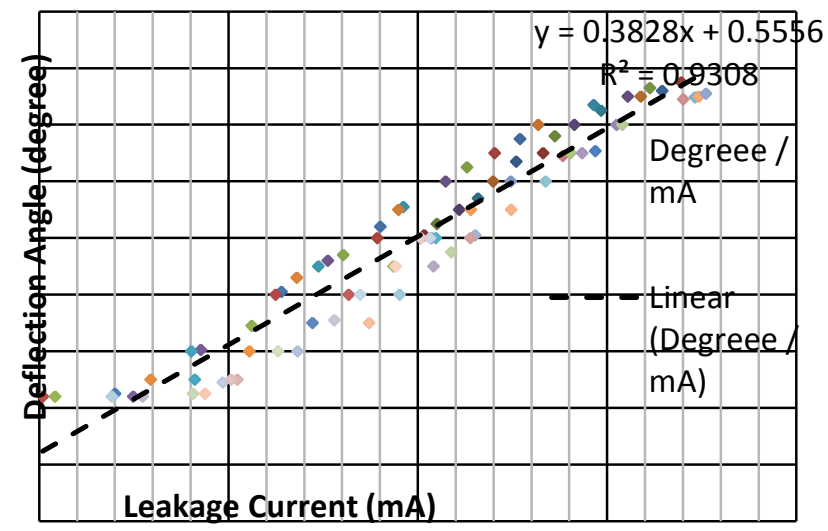

Figure 6. Measurement result plot

From the experiment, it also shows the maximum leakage current is $152.2 \mathrm{~mA}$ with deflection angle as wide as 55 degree. The other direction of maximum leakage current is at $-198.1 \mathrm{~mA}$ with deflection angle -56 degree. There is shifting at zero degree position from $0 \mathrm{~mA}$ to $-1.5 \mathrm{~mA}$. It may come from the rated of magnetic hysteresis, that is a common phenomenon of using ferromagnetic material like in compass measurement. A macroscopic model has been developed for this characteristic [16].

\subsection{Remodeling resultant magnetic force}

From the experiment, it was showed that there was a deflection angle movement rather than backward forward direction as if it only has two magnetic sources like in Figure 4. It came from the resultant magnetic force from more than two vectors. There were two sources from each solenoid coils and one source from the Earth magnetic field.

Using the simulation software EJS, it was possible to simulate the vector as the movement of compass seems to be caused by other vector influences. It was modified from Magnetic Bar Field Model 
[17]. By using simulation it was possible to model the resultant magnetic forces. That figure consists of two solenoids similar to Figure 4. The earth magnetic source is represented with a permanent magnet but with slightly different magnetic behavior. It was possible to create and simulate it on EJS. It is by defining a constant uniform magnetic vector. The resulted model can be seen at Figure 7.

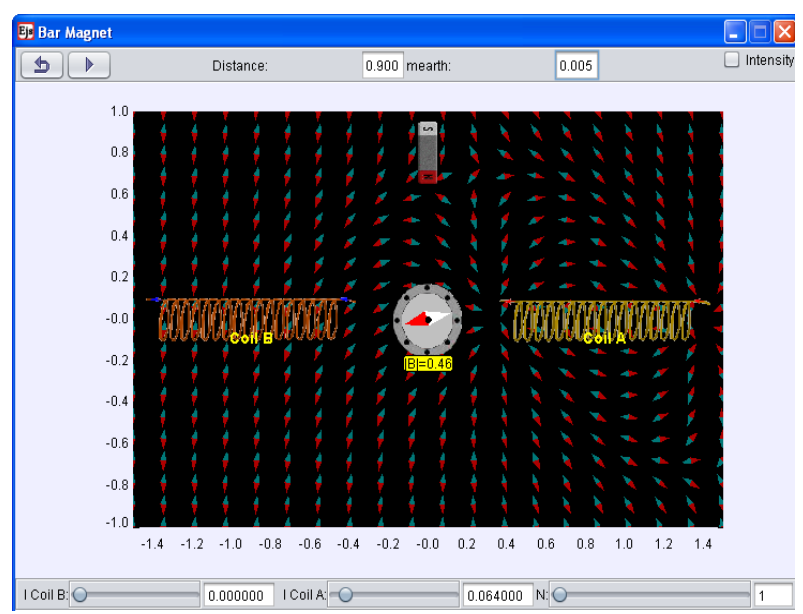

Figure 7. Remodelling experiment

The uniform vertical magnetic vector created an angle from resultant magnetic vector between the Earth magnetic field $\left(\mathrm{B}_{\text {Earth }}\right)$ and the Leakage Current Magnetic Field Difference $\left(\Delta \mathrm{B}_{\text {LeakageCurrent }}\right)$. This can be written as follows:

$$
\theta=\arctan \left(\frac{B_{\text {Earth }}}{\Delta B_{\text {LeakageCurent }}}\right)
$$

\subsection{Experiment on increasing sensitivity}

The previous result from a forementioned experiment is still showing about 0.38 degree/ $\mathrm{mA}$ and it is necessary to make feasible the possibility on increasing the measurement sensitivity. One of the proposed solution has been discussed before the simulation [8]. It is by increasing number of coil turns.

Three cases of experiment have been done to see the possibility of increasing this Experimental measurements sensitivity. It is by having different positions of the compass magnet. The cases are:

a. Single Coil at a distance from its center,

b. At center of Single Coil,

c. At center of Double Coil.

The experiment result are shown in Figure 8. The three experimental data for the Single Coil at a distance from its center, at center of Single Coil, and at center of Double Coil are represented, respectively, in blue (diamond shape), red (square) and in green (triangle). From this experiment it is seen that there is an increasing value of measurement sensitivity. The different sensitivities are $0.37,0.47$, and 0.81 (degree/mA), respectively.

The other interesting parameter on increasing sensitivity is how it correlates with the hysteresis. It is narrowing the gap. From the hysteresis shapes, it was seen that different angle values for a single leakage current is getting narrow. Intuitively it can be noticed that the increasing the sensitivity could reduce the hysteresis.

The result in Figure 8 were analyzed to get the detail calculation on how much the hysteresis is affected. The analysis were done by analyzing the relationship between the value of electric current and the deflection angle in the measurements as before.

Three conditions were considered. The first condition is to use a single source with a distance or put a magnet at a distance from the coil. The second condition is to use a single source and put the magnet in the center of the coil. The third condition is to use two sources and put the magnet in the middle of two coils. Using the hysteresis graph that has been obtained, it is now attempted to see if the sensitivity measurement 
has correlation with the hysteresis. To do that, it was necessary to characterize the hysteresis curve. As the hysteresis curve is a non-linear curve, the solution was to use two parallel lines [18]. That is, with one line passing through positive path condition and the other was using the negative path condition. The midpoint of the hysteresis was acquired by using equation as follows:

$$
X_{m}=\left(\frac{X_{\max }-X_{\min }}{2}\right)+X_{\text {min }}
$$

The midpoint value $\left(\mathrm{X}_{\mathrm{m}}\right)$ from the first condition was $0.05 \mathrm{~mA}$, from the second condition was $39.60 \mathrm{~mA}$, and from the third condition was $9.40 \mathrm{~mA}$. These values are shown in Table 2. Then midpoint in $\mathrm{Y}$ coordinate was used to determine the total of hysteresis by using equation:

$$
\text { Total }_{\text {Hysteresis }}=Y_{p}-Y_{n}
$$

Total Hysteresis Value will represent the hysteresis gap. Yp is higher value, and Yn is lower value at mid point. The result of calculation (8) is shown in Table 2. Total hysteresis of the first condition is 19.05, the second condition is 17.87 , and the third condition is 15.92. It is showing that the total hysteresis is decreasing as the increasing sensitivity. It is similar to the visualization at Figure 8 as discussed before.

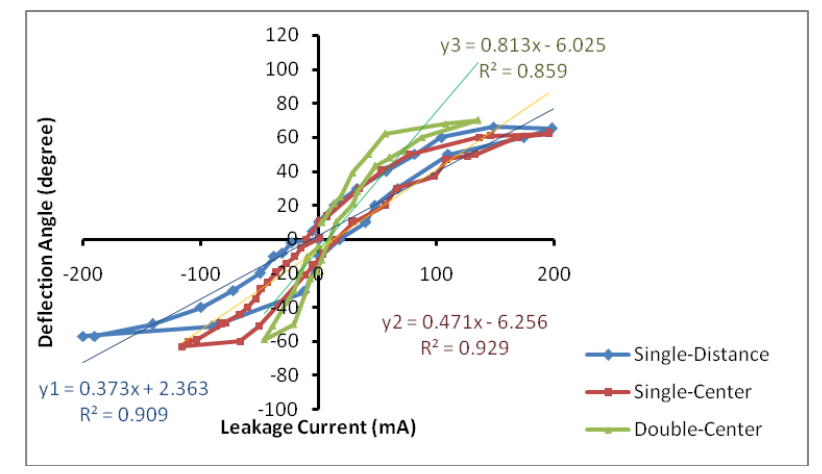

Figure 8. Increasing sensitivity

Table 2. Increasing Sensitivity

\begin{tabular}{ccc}
\hline Case & Midpoint $(\mathrm{mA})$ & Total Hysteresis $(\mathrm{deg})$ \\
\hline 1 & 0.05 & 19.05 \\
2 & 39.60 & 17.87 \\
3 & 9.40 & 15.92 \\
\hline
\end{tabular}

\section{CONCLUSION}

Compared to simulation paper [8], it is shown in this work that it is possible to have Leakage Current Measurement using Helmholtz coil configuration with different current flow as another form of RCD. The RCD Formula expressed at (3), and the Helmholtz Coil with Different Current Flow Formula expressed at (5) showed similar relation between the leakage current and the resulted magnetic field with different value of $k$ as in,

$$
\Delta B \simeq k . l_{\text {leakage }}
$$

The EJS software could be used to show the magnetic field of Helmholtz Coil with Different Current Flow. The simulation showed how the magnetic fields from two coils are cancelling each other. The other model also can follow the experiment result which has influence from the Earth Magnetic Field. A linear gradient 0.38 degree/mA could be achieved with commercial Leybold Helmholtz Coils. 
The measurement results showed the hysteresis phenomenon. However its linear equation can be achieved with high $\mathrm{R}^{2}$ value. It was as high as $93 \%$. It showed a good linearity with little variance. From the last experiment, it was seen that there was high possibility to increase the measurement sensitivity in order to achieve the standard leakage current limit for electrical devices. It showed that the hysteresis was possible to be reduced as the measurement sensitivity increased. This work still used current source power supply which comes along with the commercial Helmholtz Coil from Leybold [15]. The future work will be investigating how is the application of this low cost leakage current measurement, with AC supply and load.

\section{ACKNOWLEDGEMENTS}

This work was supported by Faculty of Science and Technology, Airlangga University for Young Lecturer's Research Fund Program. Thanks for all the team's member for the supports.

\section{REFERENCES}

[1] R. Djekidel and D. Mahi, "Capacitive Interferences Modeling and Optimization between HV Power Lines and Aerial Pipelines," International Journal of Electrical and Computer Engineering (IJECE), vol. 4, no. 4, 2014.

[2] N. A. M. Hasni, et al., "Investigation of Potential Grounding Compound for Portable Applications," International Journal of Electrical and Computer Engineering (IJECE), vol. 7, no. 6, 2017.

[3] J. Withfield, "The Electricians Guide Book," 16th edition, EPA Press, 2008.

[4] T. Kaewgun, et al., "Multi-function Electrical Safety Tester for Medical Equipment Application," 2016 International Conference on Biomedical Engineering (BME-HUST), pp. 57-60, 2016.

[5] A. Benabdellah, et al., "New Electromagnetic Force-Displacement Sensor," International Journal of Electrical and Computer Engineering (IJECE), vol. 6, no. 2, 2016.

[6] H. Zenkner and K. Werachet, "Energy Transfer by Resonance Coupling," International Journal of Electrical and Computer Engineering (IJECE), vol. 3, no. 5, 2017.

[7] Wee L. K. and Ning H., "Vernier caliper and micrometer computer models using Easy Java Simulation and its pedagogical design features--ideas for augmenting learning with real instruments," Phys. Educ., vol. 49, no. 5, 2015 .

[8] E. Sutanto, et al., "Simulation of Leakage Current Measurement in Medical Devices Using Helmholtz Coil Configuration With Different Current Flow," Journal of Physics: Conference Series, vol. 853, 2017.

[9] IEC 60364-4-41, "Protection Against Electric Shock," Geneva, International Electrotechnical Commission, 2005.

[10] IEC 60898-1, "Electrical Accessories - Circuit-Breakers For Overcurrent Protection For Household And Similar Installations - Part 1: Circuit-Breakers For A.C. Operation," Geneva, International Electrotechnical Commission. 2nd Edition, 2015.

[11] D. Kendal, "Development of high permeability cores for earth leakage protection devices," Telcon Ltd., UK. 5th International Conference on FACTORY 2000, 1997.

[12] W. Lewin and B. John, "Physics II Electricity and Magnetism," MIT OpenCourseWare, Spring, 2007.

[13] F. Hwang and W. Christian, "Helmholtz Coils," 2009.

[14] Trout S. R., "Use of Helmholtz coils for magnetic measurements," in IEEE Transactions on Magnetics, vol. 24, no. 4, 1988.

[15] Leybold, "Helmholtz Coils-555571,” Manual Book, 1999.

[16] V. F. Lavet, et al., "Vectorial incremental nonconservative consistent hysteresis model," Proceedings of the 5th International Conference on Advanded Computational Methods in Engineering (ACOMEN2011), Liege, Belgium, ACOMEN2011, 2011.

[17] W. Christian, et al., "Magnetic Bar Field Model," 2009.

[18] Mitchell S. C., "Explanation of hysteresis calculation," ME 240 - Mechanical Instrumentation, Experiement resources and supplimental information, 2011.

\section{BIOGRAPHIES OF AUTHORS}

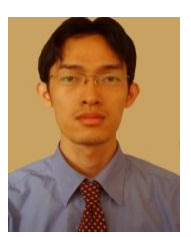

Erwin Sutanto received his BSc. in Electronics from Bandung Institut of Technology, and MSc. in IC Design (Electronics) from Nanyang Technological University Singapore in 2002 and 2008 repectively. After working for a few years in various industries, he is joining Airlangga University as a Lecturer in 2016. His research interests are in electronic circuits, electro-magnetic, and its applications in medical instrumentations. 


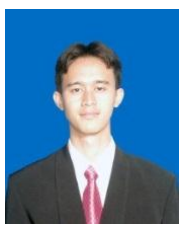

Franky Chandra graduated his Bachelor in Physical Engineering from SepuluhNopember Institute of Technology in 2016, and graduated his Master program in Electrical Engineering from SepuluhNopemberInstitut of Technology in 2008. He has been joining Airlangga University since 2009 as a Lecturer. His research interests are in electronics and instrumentation, computation, image processing, and robotics.

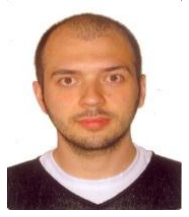

Eduardo Gonnelli obtained his Bachelor degree in Medical Physics at the São Paulo State University "Julio de Mesquita Filho" (UNESP- Brazil) in 2010. He obtained the Master degree in Nuclear Technology- Reactors at the Nuclear and Energy Research Institute (IPEN/CNEN - Brazil) in 2013. He obtained his Ph.D degree in Nuclear Technology- Reactors at the Nuclear and Energy Research Institute (IPEN/CNEN - Brazil) in 2017. His research interests are Nuclear Reactor Physics, Numerical Analisys, Signal Processing and Experimental Data Acquisition.

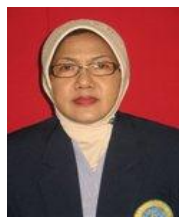

Suhariningsih, obtainedher Bachelor of Engineering Physics from SepuluhNopember Institute of Technology in 1978. She joinedas a lecturer inAilangga University since 1978. Then she obtained her Ph.D. Degree in Biophysics from Airlangga University in 1999. Her research interests are Biophysics, Medical Physics, and Bioelectromagnetism. She has a Patent Akupotensiograf. 